st $40^{\circ}$ in 15 minutes, and a full colour intensity only at $80^{\circ}$ in the same time. Probably the promoting effect of sodium fluoride on the colour intensity is due to accumulation of ketose in the yeast. According to the findings of Jordan and Pryde, it is more likely a question of a hexosemonophosphoric or hexosediphosphoric acid accumulation than that of a triose.

In the case of yeast dried at room temperature, the drying process seems to play a similar part to the addition of sodium fluoride.

Experiments seem to show that in the case of ketoses the condensation with tryptophane commences at $90^{\circ}$, without the presence of a strong acid. Therefore in the case of red dried yeast, too, the assumption of ketose accumulation is a very probable explanation.

That the carbohydrate metabolism is disturbed by sodium fluoride is well known. In the presence of added substrate there is an accumulation of phosphoglyceric acid ${ }^{5,6,7}$. Without added substrate-as in my experiments - there is an accumulation of hexose monophosphoric acid in dried brewer's yeast ${ }^{5}$, hexose diphosphoric acid in dried plesmolysed baker's yeast8. There seem to be no reports of possible accumulation products in the absence of added substrate in fresh bakers' yeast, for which reason the above-mentioned observations might afford an in. sight into related problems. On the basis of the observations of Jordan and Pryde, a sensitive colourreaction for ketoses could be developed with the yeast substances as reagents. The quantitative determinations which have been made indicate that such a method would render it possible to follow, in a simple manner, even smell variations in the quantity of ketoses in the yeast under varying conditions.

Wenner-Gren's Institute for Mignon Malm.

Experimental Biology,

University of Stockholm. April 4.

'Bardachzi, F., Hoppe-Seylers Z., 48, 145 (1906)

- Jordan, C., and Pryde, J., Biochem. J., 32, 279 (1938).

'Ohyama, Y., J. Biochem., 27, 351 (1938).

- Karaoglanov, Z., Z. analyt. Chem., 121, 92 (1941).

silsson, R., Sv. Vet. Ak. Ark. Kemi, Mineral. och Geol., A, 10, No. 7 (1930).

- Neuberg, C., and Kobel, M., Biochem. Z., 263, 221 ; 264, 456 (1933).

'Meyerhof, O., and Kiessling, W., Biochem. Z., 267, 331 (1933),

'Myrbäck, K., and Ortenblad, B., Biochem. Z., 292, 230 (1937).

\section{Halogenation in the Allyl Position}

We should like to make a few comments on the recent communication by W. A. Waters ${ }^{2}$ in criticism of F. S. Spring ${ }^{2}$ for stating that substitution in the allyl position of olefines is a new reaction attributable to Ziegler and his collaborators ${ }^{3}$.

Although the reactions of halogen-containing substances with olefines to effect halo-substitution on allyl carbon atoms are of great interest, it seems to us that the most important reaction of allylic substitution is the direct halogenation of olefines at elevated temperatures discovered by Groll and others" in these Laboratories. Thus, for example, at tem. peratures of the order of $400^{\circ} \mathrm{C}$., by an apparently homogeneous reaction, almost all the halogen in a $1: 7$ mixture of chlorine and propylene reacts readily by substitution to form allyl chloride. Bromine behaves similarly.

Waters conveys the impression that Farmer and his colleagues ${ }^{5}$ were the first to show that the substitution into olefines in the $\alpha$-methylene position was a reaction involving free radicals. In the reference to Farmer, cited by Waters, that author himself attributes the interpretation of the mechanisms of high temperature substitutive halogenation of olefines to two of the authors of this letter. It has been shown that both addition and substitution occur under the experimental conditions principally, although not exclusively, by radical chain mechanisms, and that substitution is the dominant reaction at higher temperatures. Catalysis of the chain processes was achieved by the use of small amounts of tetra-ethyl lead, and, while under some conditions oxygen was a powerful suppressant of both reactions, under others a trace of oxygen acted as a powerful catalyst for the substitutive chlorination of propylene to form allyl chloride at temperatures as low as $272^{\circ} \mathrm{C}$. In this connexion it may be mentioned that Ingold ${ }^{7}$ states that peroxide catalysis (and hence by analogy oxygen catalysis) of a reaction is "compelling" evidence for a free radical mechanism. Such evidence is also accepted by Waters" as "conclusively" proving that such a process occurs. Of course, there is a wealth of experimental evidence for both acceleration and inhibition of radical reactions, from which may be cited Kharasch's oxygen- and peroxide-catalysed 'abnormal' addition of hydrogen bromide to unsymmetric olefines ${ }^{9}$ and the quantitative photochemical 'anti-Markownikov' reaction involving such olefines and either hydrogen bromide or hydrogen sulphide ${ }^{10}$.

As have others in the past ${ }^{11}$, we wish to emphasize that the postulation ${ }^{12}$ of free hydrogen atoms, produced by attack of organic radicals on molecules, as chain-carrying species in reactions is precarious, as the energetics of such reactions are generally entirely unfevourable.

WHIIAM E. VAUGHAN. Freiderick F. Rust. George W. Hearne.

Laboratories of

Shell Development Company, Emeryville, California. March 3.

Nature, 154, 772 (1944).

2 Annual Reports of the Chemical Society, 40, 101 (1943).

- Annalen der Chemie, 551, 80 (1942).

- Groll and Hearne, Ind. Eng. Chem., 81, 1530 (1939). Engs and Redmond, U.S. Patent 2,077,382, April 20, 1937. Groll, Hearne,

Trans. Faraday Soc., 38, 340 (1942).

- J. Org. Chem., 5, 472 (1940).

Trans. Faraday Soc., 37, 791 (1941).

- Trans. Faraday Soc., 37, 792 (1941).

- See Mayo and Walling, Chem. Rev., 27, 351 (1940).

10 Vaughan and Rust, J. Org. Chem., 7, 472, 477 (1942).

is See, for example, ref. (9), (p. 358); also, Weiss, Trans. Faraday Soc., 37, 793 (1941).

12 For example, see references (5) and (8).

As Spring noted in his report, due credit must be given to the chemists of the Shell Development Company of California for their original success in effecting the vapour-phase halogenation of olefines in the allyl position. While their evidence for the atomic mechanism of this process might have received mention, there can now be fow chemists who would picture a homogeneous gas reaction of this type as anything other than a free radical process.

The mechanism of chlorine substitution in solution is, however, less well established, and in the 